\title{
Bifid Hyperplastic Mandibular Condyle
}

\author{
R. S. Neelakandan · Darpan Bhargava
}

Received: 18 March 2011 / Accepted: 30 June 2011/Published online: 27 July 2011

(C) Association of Oral and Maxillofacial Surgeons of India 2011

\begin{abstract}
Condylar hyperplasia is a rare non-neoplastic pathology associated with overgrowth of the mandibular condyle. Presentation of condylar hyperplasia with bifid mandibular condyle has never been reported in literature. Early management of the hyperplastic disorders of the mandibular condyle can prevent occlusal canting and developing asymmetric deformities. We report a case of 'Bifid Hyperplastic Mandibular Condyle' in a 14-year-old male with emphasis on early surgical intervention. To best of our knowledge, the present case is the first reported case of bifid mandibular condyle with condylar hyperplasia and 66th reported case of bifid mandibular condyle in living human population.
\end{abstract}

Keywords Condylar hyperplasia · Bifid condyle .

Mandibular condyle $\cdot$ Hyperplastic mandibular condyle

\section{Introduction}

Condylar hyperplasia is a rare non-neoplastic pathology associated with overgrowth of the mandibular condyle [1]. This disorder is the most common post-natal growth abnormality affecting the temporomandibular joint [2]. The overgrowing condyle can result in facial asymmetry, mandibular deviation, malocclusion, and articular dysfunction [3]. Condylar Hyperplasia usually presents as a developing mandibular asymmetry which manifests either during the pubertal growth spurt as a result of an abnormal

R. S. Neelakandan · D. Bhargava $(\square)$

Department of Oral and Maxillofacial Surgery, Meenakshi

Ammal Dental College and Hospital, Alapakkam Main Road,

Maduravoyal, Chennai 600095, India

e-mail: emaildarpan@gmail.com hypermetabolic growth centre in the affected condyle, or at the end of puberty when growth in one condyle persists into adulthood [4]. We report a rare case of a bifid mandibular condyle with condylar hyperplasia.

\section{Case Report}

A 14-year-old male patient reported to our oral and maxillofacial unit with a chief complaint of asymmetric face with deviated chin. On clinical evaluation, we observed that the vertical facial thirds and transverse facial fifths were within normal limits except for an obvious midline deviation of the lower third of the face towards the left side (Figs. 1, 2). The mouth opening was adequate with normal excursive and protrusive movements. The patient had Angle's class III molar relationship on the left side and Angle's class I on the right side. Maxillary arch had a mesiodens and the mandibular midline was shifted towards the left side by $7 \mathrm{~mm}$.

\section{Radiographic Evaluation}

PA Skull projection showed clear midline deviation of the mandibular midline towards the left side with elongated mandibular condyle-ramal unit on the right side. Occlusal cant was not significant (Fig. 3).

Panoramic radiograph demonstrated an interesting finding of the elongated right mandibular neck dividing proximally to form two condylar heads. The mandibular foramen on the right side was ill-defined when compared to the left side of the mandible. The right inferior dental canal cortices were not as prominent as on the left side and the right canal was at a lower level than the left side (Fig. 4). 

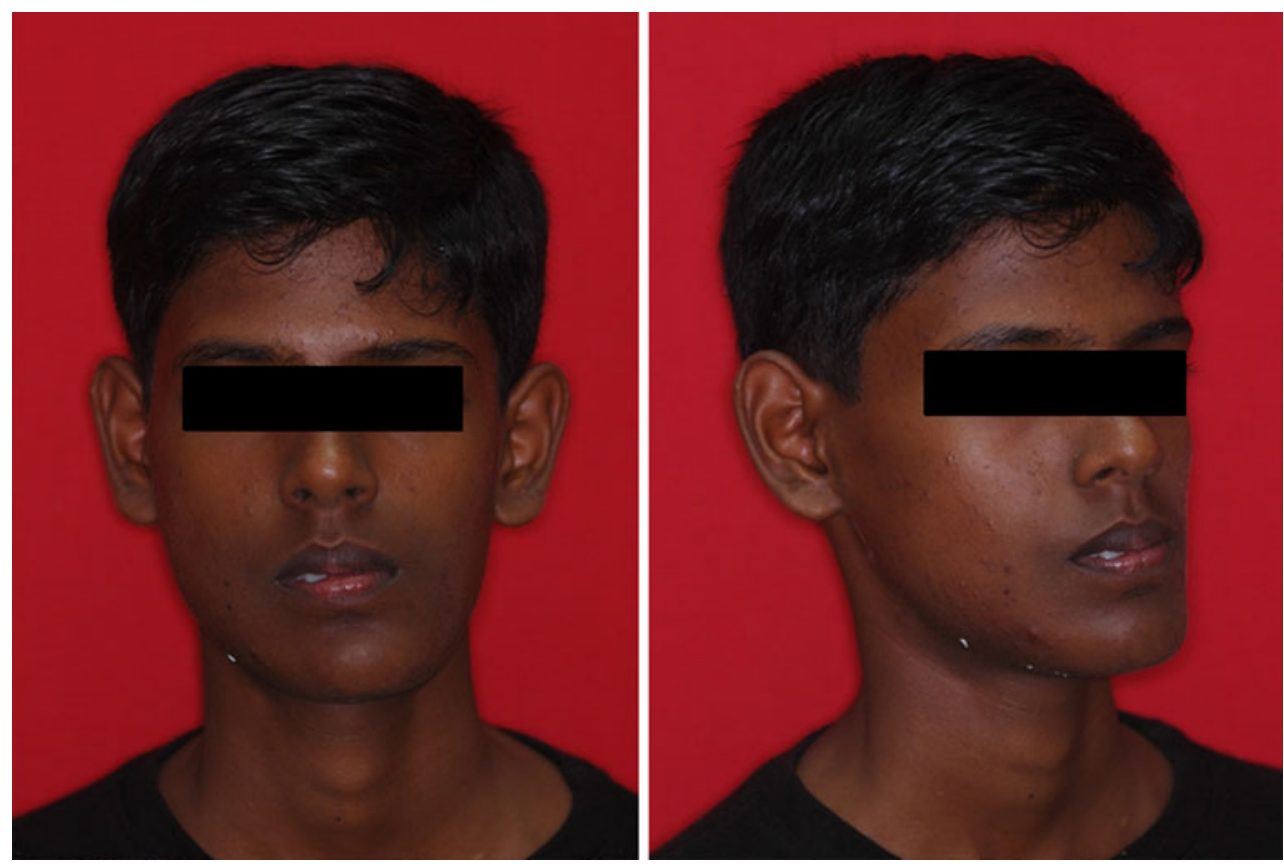

Fig. 1 Patient with midline deviation of the lower third of the face towards the left side

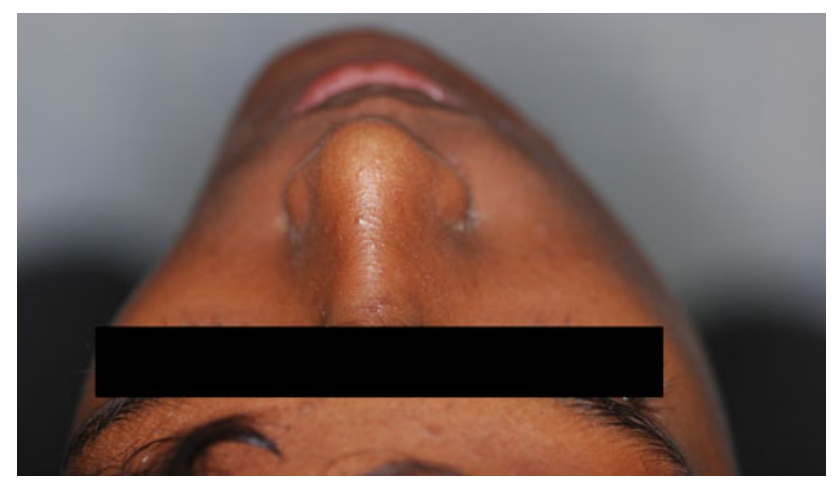

Fig. 2 Bird's eye view showing the midline deviation

Computed tomography scan showed similar findings of an elongated right mandibular condylar neck. The right mental foramen was located at a lower level. Scan showed a clear bifid right mandibular condyle (Fig. 5).

Hand wrist radiograph was taken to assess the skeletal age and maturation, more importantly to assess the percentage of the residual maxillary and mandibular growth using Fishman's skeletal Maturity Index. Capping of the epiphysis was seen in the middle phalanx of the fifth finger classifying the hand wrist radiograph as skeletal maturity index (SMI) 7. The index gave us the approximate chronological age of $14.38 \pm 1.08$ years, with $69 \%$ of maxillary growth and $66.7 \%$ mandibular growth completed (percentage of maxillary growth remaining, $31 \%$ and mandibular growth remaining, 33.3\%) (Fig. 6).

Tc-99m methylene diphosphonate (MDP) bone scintigraphy was performed following standard protocols after

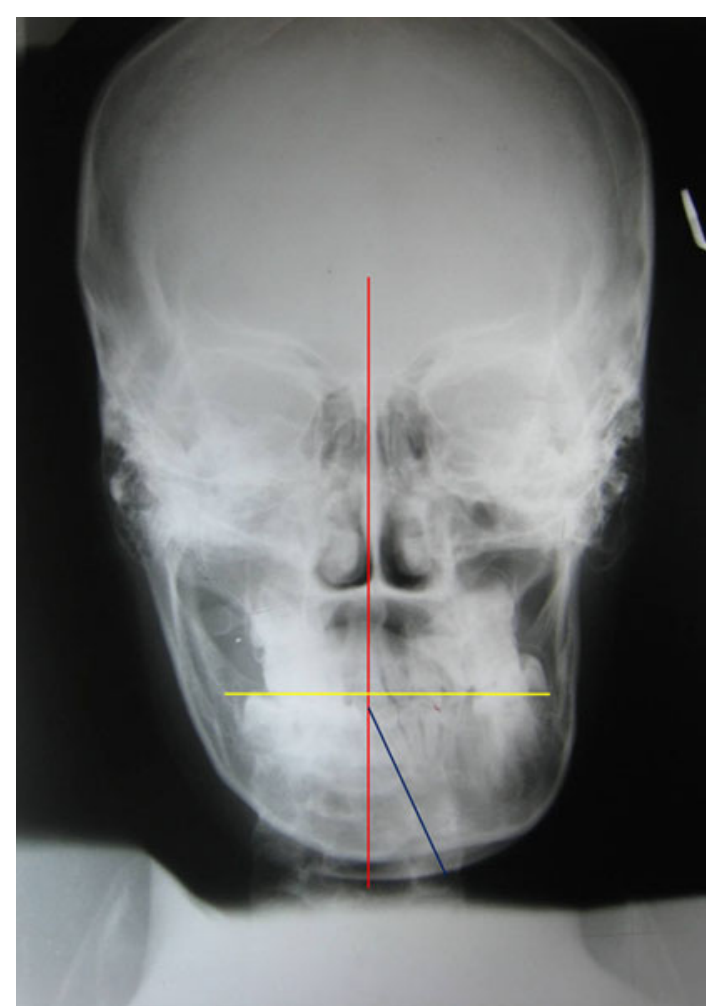

Fig. 3 PA Skull projection with midline deviation of the mandibular midline towards the left side with elongated mandibular condyleramal unit on the right side

I.V. administration of $20 \mathrm{~m} . \mathrm{Ci}$ activity to assess the condylar growth. There was an increased tracer concentration in the condylar process of the right mandible suggestive of 


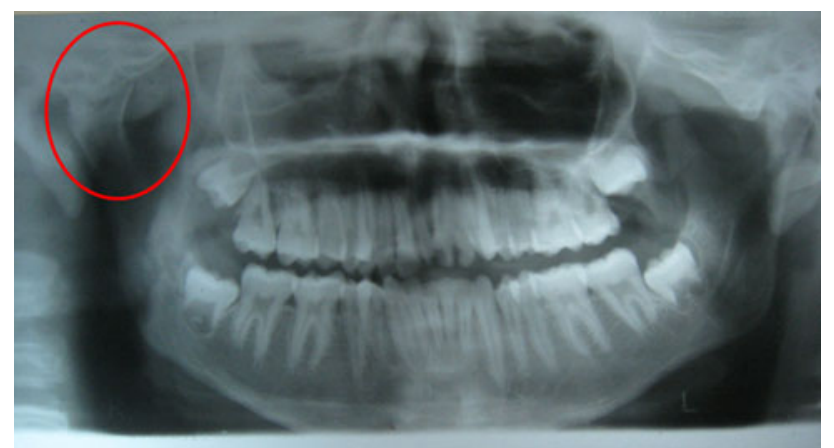

Fig. 4 Panoramic radiograph of the patient

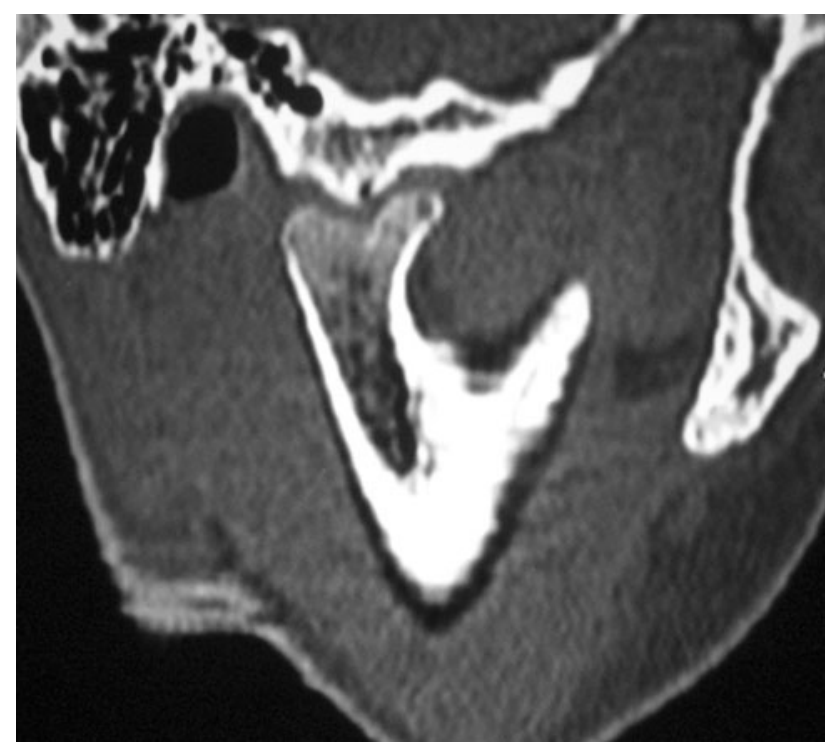

Fig. 5 Computed tomography scan showed a bifid right mandibular condyle

hypermetabolic active bone growth and ossification process. The ramus of the right mandible appeared relatively elongated. The tracer concentration in left condylar region and the rest of the skeletal system appeared to be within physiological limits (Fig. 7).

Developing mandibular asymmetry and scintigraphy proven abnormal hypermetabolic growth in right mandibular condyle directed us towards a working diagnosis of right condylar hyperplasia. A right condylectomy was planned to disrupt the actively growing condylar growth center.

\section{Surgical Procedure}

The surgical treatment planned was open condylectomy under general anesthesia. The right condyle was exposed with a preauricular incision, and condylectomy was performed with chisel and mallet after making postage-stamp cortical marking with fissure bur. The resected condylectomy specimen showed a clear bifid condylar head (Fig. 8).

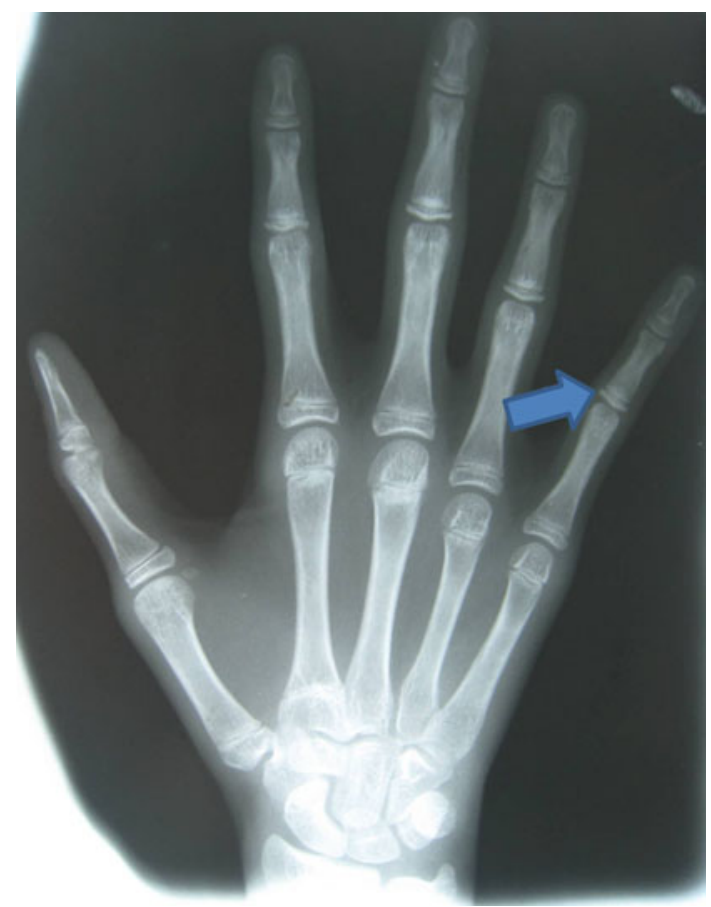

Fig. 6 Hand wrist radiograph to assess the skeletal age and maturation. Capping of the epiphysis is seen in the middle phalanx of the fifth finger classifying the hand wrist radiograph as skeletal maturity index (SMI) 7

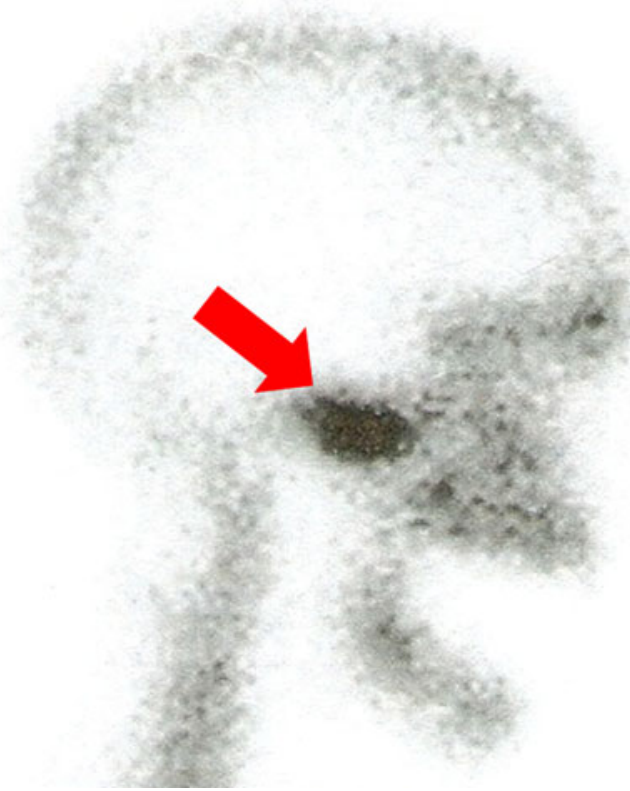

Fig. 7 Tc-99 m methylene diphosphonate (MDP) bone scintigraphy with increased tracer concentration in the condyle of the right mandible suggestive of hypermetabolic active bone growth and ossification process

The condylar stump was smoothed with a bone file and a round bur. Great care was taken to prevent injury to the facial nerve. The disc was identified and was secured over 


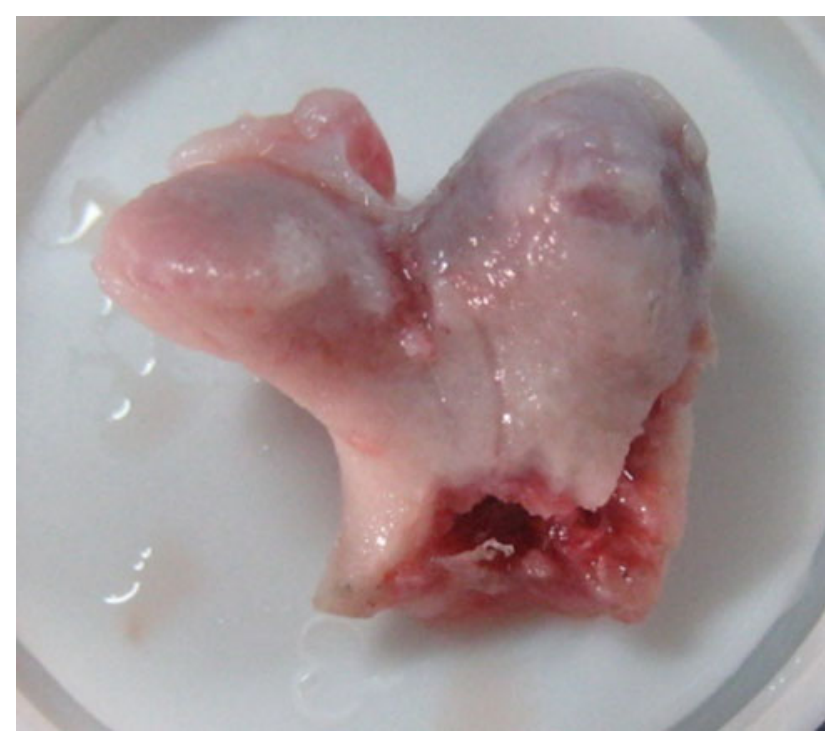

Fig. 8 Resected condylectomy specimen

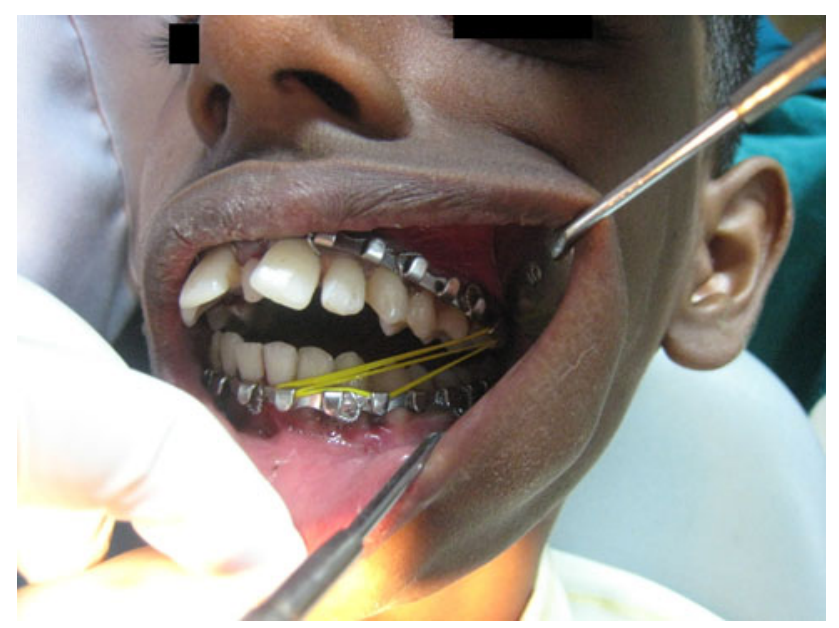

Fig. 9 Class III elastic traction to guide and settle the occlusion for 1 week in the post operative period

the neo-condyle. In the immediate postoperative period the patient developed left sided open bite (contralateral side to surgery) and premature contact of occlusion on the right side due to shortened right condylar process. The patient was put on class III elastic traction on the left side with the help of arch bars fixed in both upper and lower arches to guide and settle the occlusion for 1 week in the post operative period (Fig. 9). A guiding flange appliance was fabricated to guide the mandible and train the temporomandibular apparatus to adapt to the desired new occlusion (Fig. 10). A 6-month period of active physiotherapy was instituted to rehabilitate the joint. The post operative panoramic radiograph showed readaptation of the right condyle to the glenoid fossa and clinically no TMJ dysfunction during first 6 months of the follow-up visits (Fig. 11). The patient discontinued the use of guiding flange appliance after 3 months in the post operative period and to our surprise there was dental and facial midline correction with adequate mouth opening and bilateral Class I molar occlusion (Figs. 12, 13). The patient was referred to the department of orthodontia for minor refinement of occlusion but the treatment was discontinued due to patient's non-compliance.

\section{Discussion}

Condyar hyperplasia was first described by Adams in 1836 as a disorder causing overdevelopment of the mandible [5]. The etiology of condylar hyperplasia is controversial. Hormonal influences, hypervascularity, heredity, infection, or trauma has been suggested by various authors as triggering factors, although no such history was elicited in the presented case [6]. It has been suggested that the clinical picture varies depending upon the age of onset of the condition or the stage of the disorder [4]. Presenting signs and symptoms include unilateral condylar elongation, temporomandibular joint (TMJ) pain and dysfunction, facial asymmetry, chin deviation, mandibular prognathism, posterior crossbite and open bite, canting of the occlusal plane, masticatory dysfunction, and malocclusion [3]. Radiologically, Obewegeser and Makek reported that the condyle appears enlarged and that its head is usually irregular and deformed and its neck thickened and elongated, with coarse trabeculae filling the condyle in cases of condylar hyperplasia [7]. The mandibular angle is typically round, with the canal in a low position. Joint pain was reported in $70 \%$ of the patients [8]. Most of these radiological findings were apparent in the presented case. Technetium 99 scintiscanning is an essential diagnostic tool. The radioactive isotope used for bone scanning is technetium 99 methylene diphosphonate. Scintiscanning is useful for the following reasons: it is possible to identify the affected joint, it becomes evident whether there is an abnormal condylar growth centre or whether there is generalized mandibular overgrowth, and it is also apparent whether or not the hyperplasia is still active or if it has become stable [4]. The hand wrist radiographs does not have a major role in predicting the growth pattern of the affected aberrantly growing hemimandible/mandible, but will give an approximate estimate of the remaining maxillary growth and therefore predictability of the chances of the maxillary canting. Fishman's skeletal Maturity Index put forth by Leonord and Fishman in 1982 is a widely followed method and was used for the presented case. Skeletal maturity index showed 69\% of maxillary growth completion, with $31 \%$ remaining growth that can result in the development of the maxillary cant and worsen the deformity. The histological features of condylar hyperplasia are generally accepted as being the 

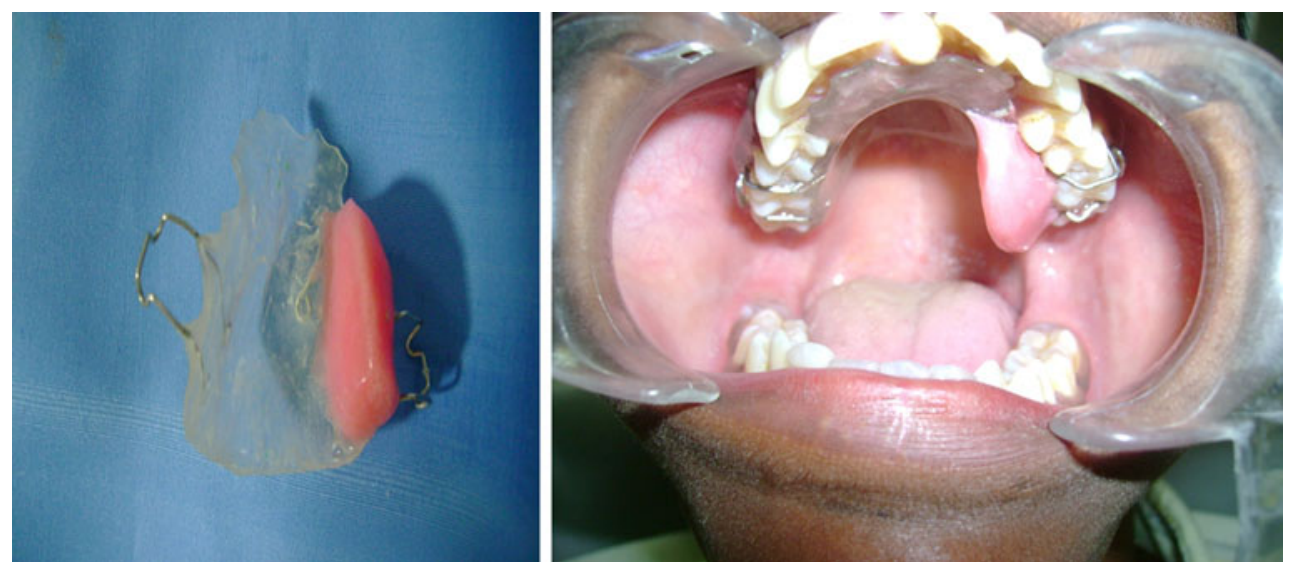

Fig. 10 A guiding flange appliance to guide the mandible and train the temporomandibular apparatus to adapt to the desired new occlusion

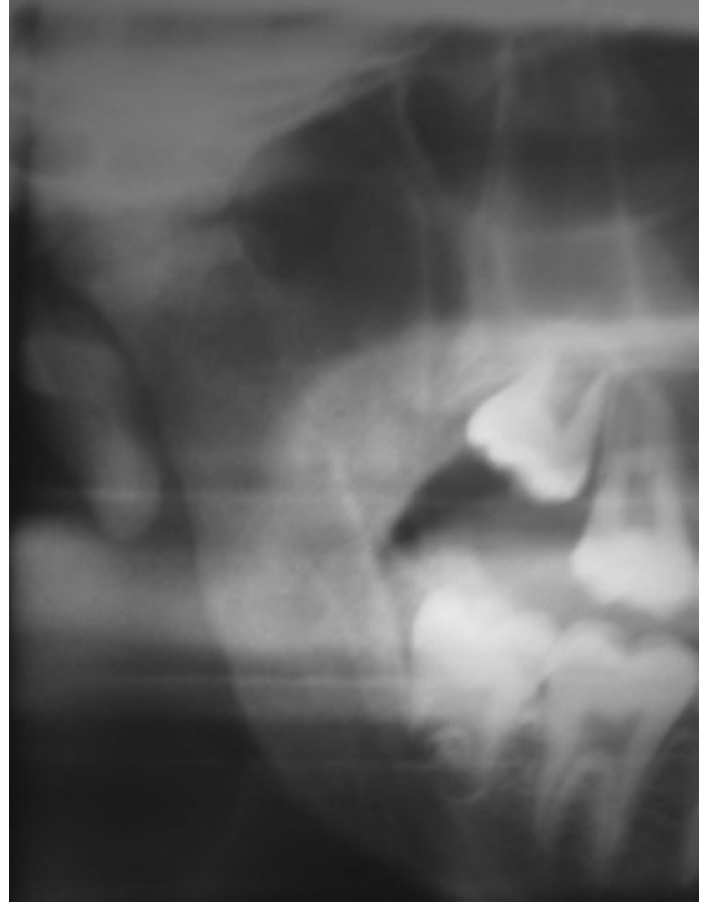

Fig. 11 Post operative panoramic radiograph showing functional condyle at 6 month follow up

presence into adulthood of a continuous germinative layer of undifferentiated mesenchyme cells, a hypertrophic cartilage layer and the presence of cartilage remnants or "islands" in the bony trabeculae [4]. In our case the histopathologic impression was hypertrophic cartilaginous layer with inflammatory changes in the joint.

In literature, this disorder has been described as a selflimiting condition, but as long as it remains active, the asymmetry progresses with the associated occlusal changes [9]. This progressing deformity warrants early recognition and an early resolution of the problem. The growth of the affected hemimandible leads to a deviation of the mandible

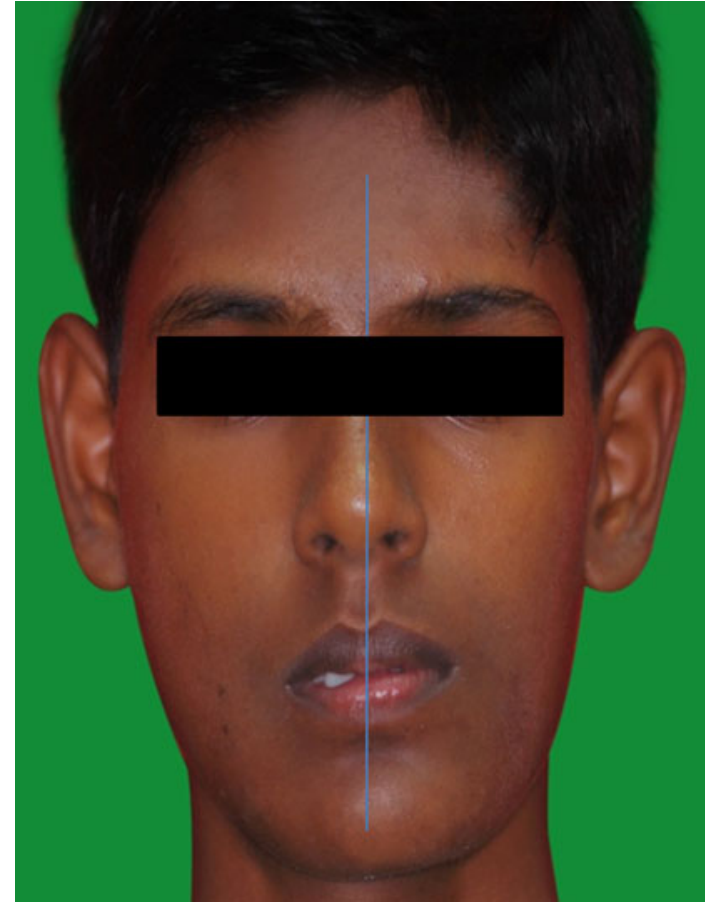

Fig. 12 Facial midline correction at 6 month follow-up

towards the unaffected side. To compensate for the complex geometrical misalignment during growth of the lower jaw, the maxillary plane is tilted. The operations are usually therefore intended to correct the dental and skeletal asymmetry and to remove the growth centre by the bimaxillary operation [6]. But if this condition is recognized early before the maxillary plane is tilted, high condylectomy should be the preferred surgical option in cases of scintigraphically proven actively growing condyles and the abnormally growing condyle should be removed at the earliest to prevent further deformity and thereby preventing the maxillary canting. It has been reported in literature that the treatment of hemimandibular 


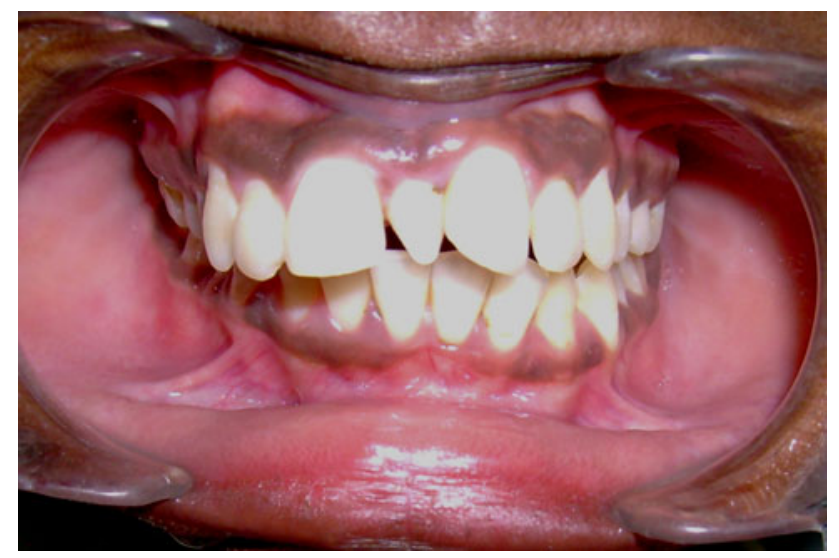

Fig. 13 Dental occlusion at 6 month follow-up

hyperplasia with combined orthodontic-maxillofacial treatment including condylectomy produces good aesthetic and functional results [6]. Condylectomy should be performed as early as possible. The reason for early treatment is to prevent the development of an oblique occlusal plane and other asymmetric deformities. Spontaneous remodeling processes in the facial structures after elimination of an actively growing hyperplastic condyle may bring about improvement in symmetry [10] or simplify treatment in the future by modifying the unfavorable growth pattern [11].

Bifid mandibular condyle is a rare condition characterized by a division of the mandibular condylar head. Review of literature by O Miloglu et al. suggests only 65 cases of bifid mandibular condyle in living population [12]. The presented case will be the 66th reported case of Bifid mandibular condyle in a living individual. The etiology of bifid condyle is largely unknown, although various factors have been suggested as possible causes like endocrine disturbances, exposure to teratogens, nutritional deficiencies and, infection and radiation [13]. It is also claimed that the condyle divides because of an obstructed blood supply during its development [14]. Blackwood stated that the condylar cartilage during the early stages of development is divided by well-vascularized fibrous septa. He suggested that persistence of this type of septum in exaggerated form within the growing cartilage might lead to an error in development [15]. But existence of such a septa is controversial. Gundlach et al. believed that the bifid condyle is a form of embryopathy caused by a combination of a teratogenic agent and misdirection of muscle fibres, which then influences bone formation [16]. Further studies are necessary to understand the etiology of a bifid mandibular condyle.

\section{Conclusion}

To best of our knowledge, the present case is the first reported case of bifid mandibular condyle with condylar hyperplasia. The PubMed Database search did not show any results for "bifid hyperplasic condyle" and "bifid hyperplasic mandibular condyle". The presented case will be the 66th reported case of bifid mandibular condyle in a living individual. Condylectomy is one of the various treatment protocols described for condylar hyperplasia, but it is expected that the removal of the condyle will stop the growth of the mandible and can therefore provide stable long-term results. Early treatment can prevent the development of an oblique occlusal plane and other asymmetric deformities.

Conflict of Interest None.

\section{References}

1. Nitzan D (2009) BAOMS annual scientific meeting, Bournemouth, 3-5 June 2009 symposium: the management of mandibular asymmetry. Br J Oral Maxillofac Surg 47:502-504

2. Kaban LB (1988) Congenital and acquired growth abnormalities of the temporomandibular joint. In: Keith DA (ed) Surgery of the temporomandibular joint. Blackwell Scientific, London, pp 67-75

3. Motamedi MHK (1996) Treatment of condylar hyperplasia of the mandible using unilateral Ramus osteotomies. J Oral Maxillofac Surg 54:1161-1169

4. Gray RJM, Sloan P, Quayle AA, Carter DH (1990) Histopathological and scintigraphic features of condylar hyperplasia. Int J Oral Maxillofac Surg 19:65-71

5. Wolford LM (2002) Efficacy of high condylectomy for management of condylar hyperplasia. Am J Orthod Dentofacial Orthop 121:136-151

6. Lippold C (2007) Treatment of hemimandibular hyperplasia: the biological basis of condylectomy. $\mathrm{Br} \mathrm{J}$ Oral Maxillofac Surg 45:353-360

7. Obwegeser HL, Makek MS (1986) Hemimandibular hyperplasiahemimandibular elongation. J Maxillofac Surg 14:183

8. Hampf G, Tasanen A, Nordling S (1985) Surgery in mandibular condylar hyperplasia. J Maxillofac Surg 13:74

9. Nitzan DW (2008) The clinical characteristics of condylar hyperplasia: experience with 61 patients. J Oral Maxillofac Surg 66:312-318

10. Ali AM, Sharawy M (1995) Enlargement of the rabbit mandibular condyle after experimental induction of anterior disc displacement: a histomorphometrlc study. J Oral Maxillofac Surg 53:544

11. West R (1999) Hemimandibular hyperplasia and elongation. J Craniomaxillofac Surg 22:10

12. Miloglu O et al (2010) The frequency of bifid mandibular condyle in a Turkish patient population. Dentomaxillofac Radiol 39:42-46

13. Quayle AA, Adams JE (1986) Supplemental mandibular condyle. Br J Oral Maxillofac Surg 24:349-356

14. Hrdlicka A (1941) Lower jaw: double condyles. Am J Phys Anthropol 28:75-89

15. Blackwood HJ (1957) The double-headed mandibular condyle. Am J Phys Anthropol 15:1-8

16. Gundlach KK, Fuhrmann A, Beckmann-Van der Ven G (1987) The double-headed mandibular condyle. Oral Surg Oral Med Oral Pathol 64:249-253 